\title{
Dogs' Brain Electric Activity in Ethylene Glycol Poisoning
}

\author{
Paula Maria SAFTENCU, Gabriela Dumitrița STANCIU, Mihai MUSTEAȚĂ, Sorin Ioan BEŞCHEA CHIRIAC, \\ Sorin Aurelian PAȘCA, Gheorghe SOLCAN* \\ Faculty of Veterinary Medicine, University of Agricultural Science and Veterinary Medicine "Ion Ionescu \\ de la Brad" 8 Sadoveanu Alley, Faculty of Veterinary Medicine, Iaşi, Romania \\ Corresponding author: gsolcan@uaiasi.ro
}

Bulletin UASVM Veterinary Medicine 72(1) / 2015,

Print ISSN 1843-5270; Electronic ISSN 1843-5378

DOI:10.15835/buasvmcn-vm: 10520

\begin{abstract}
Ethylene glycol (EG) poisoning is one of the most common toxicosis in dogs, even a small amount being lethal if the antidote is not administered on time. Once ingested the breakdown products of the toxic cause acute renal failure. Neurological signs include dizziness, ataxia, and seizures similar to those from idiopathic epilepsy or other neurologic disorder. The aim of this study was to describe electroencephalographic changes in dogs suffering from EG toxicosis using a repeated short time electroencephalographic (EEG) recording. EEGs was performed on 5 mixed breed with ages between 4 and 9 years old suffering from EG poisoning, free of other neurologic disorders or previous seizures.

Electroencephalographic recording was obtained using five subdermal needle electrodes placed using the Redding model. The EEG was recorded with sensitivity $=70 \mu \mathrm{V} / \mathrm{cm}$; time constant $=0.3$ seconds impedance of all electrodes $<10 \mathrm{k} \Omega$. Each patient was EEG analysed at every 24 hours.

The EEG trace was normal in the initial state. With toxicosis progression different EEG anomalies were observed. The EEG traces slightly voltated presented an initially slowed background activity of 5-7 cycles/second, above which synchronous bilateral diffuse delta waves where registered, as an expression of cortical or subcortical neuronal hyperexcitability state. In advanced stages, when the patients elicit seizures clinically, the EEG recording trace became irregular and arrhythmic with epileptic abnormalities.
\end{abstract}

Keywords: dog, electroencephalogram, ethylene glycol poisoning.

\section{INTRODUCTION}

Ethylene glycol (EG) is a common household toxic found in anti-freeze, brake fluid and other industrial products, odorless and with a sweet taste; it is very often accidentally ingested by children and pets. Even though all animals are susceptible to EG poisoning it is most commonly encountered in dogs and cats, being associated with antifreeze ingestion. Symptoms may appear after consuming only a small quantity of this fluid, for an average size dog a tablespoon being enough to cause fatal kidney failure. Early symptoms may occur within 30 minutes of ingestion, but it can take even up to 12 hours for the first signs of poisoning to manifest $(1,7,12)$.

EG itself is not toxic, but due to its biotransformation in the liver in the presence of alcohol dehydrogenase, toxic metabolites are formed that cause metabolic disorders and renal damage. Being dependent on the doses and the time elapsed after ingestion the clinical signs can be divided in those caused by non-metabolized EG and those cause by its breakdown products $(3,7)$.

After ingestion EG is completely absorbed from the gastro-intestinal (GI) tract in a period of one to three hours, this is when the first signs of poisoning start showing that resemble alcohol intoxication, the animal may look inebriated, ataxic or simply become inactive $(7,12)$.

Eventually the signs may improve, even without treatment, this is when the EG passes to liver and begins its transformation in glycoaldehyde via alcohol dehydrogenase, other major metabolites are than formed such as glycolic, 
glyoxylic, and oxalic acid that lead to an anionic gap and metabolic acidosis, in this stage dogs exhibit cardiopulmonary signs, with increased heart and respiratory rate (2).

Within 36 to72 hours kidney failure accompanied by oliguria, may develop, with vomiting due to GI irritation, polydipsia and polyuria, and neurologic signs such as depression, stupor, ataxia, knuckling, decreased withdrawal and righting reflexes and even seizures, similar to those from idiopathic epilepsy or other neurologic disorder $(1,4,7)$. Kidney failure is caused by the deposition of calcium oxalate crystals formed due to the affinity that the oxalic acid has towards the calcium in the dog`s blood.

Early diagnostic and therapeutic intervention is very important to assure a favorable outcome.

The aim of this study was to describe electroencephalographic changes in dogs suffering from EG toxicosis using a repeated short time electroencephalographic (EEG) recording in order to eliminate other neurologic disorder such as epilepsy, as the cause of the of the seizures and the other neurologic signs.

\section{MATERIAL AND METHODS}

EEGs was performed on 5 mixed breed with ages between 4 and 9 years old suffering from EG poisoning, free of other neurologic disorders or previous seizures.

The research was conducted in the Internal Medicine Clinic of the Faculty of Veterinary Medicine Iasi.

Ethylene glycol poisoning was diagnosed based on the case history from the owner, clinical examination and blood work using Cormay Accent chemistry analyzer; complete blood cell count was done using vet $\mathrm{ABC}$ Hematology Analyzer; ultrasound imaging was obtained using Aquila ProVet ultrasound machine from Esaote Piemedical.

All patients were sedated before EEG recording, using medetomidine hydrochloride $0.03 \mathrm{mg} / \mathrm{kg}$ inj. i.m., to eliminate the artefacts caused by the muscle contractions, after the electroencephalographic examination saline solution was administered IV to eliminate the sedative.

Electroencephalographic recording was obtained using five subdermal needle electrodes (F3, F4, 01, $\mathrm{O} 2$ and $\mathrm{Cz}$; disposable subdermal stainless steel EEG needle) placed using the Redding model, the area where the electrodes where places was degreased with alcohol.

The reference electrode was placed on the bridge of the nose and the ground electrode right caudal to the occipital protuberance. The EEG (the Neurofax S, MEB 9400K Nihon Kohden) was recorded with sensitivity $=70 \mu \mathrm{V} / \mathrm{cm}$; time constant $=0.3$ seconds; $\mathrm{Hf}=70 \mathrm{~Hz} ; \mathrm{Lf}=0.5 \mathrm{~Hz}$; notch filter inserted; impedance of all electrodes $<10 \mathrm{k} \Omega$.

The signals were analog filtered to eliminate the "background noise" caused by signals from the mains frequency 50 to $60 \mathrm{~Hz}$ and other "urban" frequencies, frequencies above $40 \mathrm{~Hz}$ were removed, and those in the range $50-60 \mathrm{~Hz}$; also the low frequency determined by physiological bio potentials (e.g. breathing).

All EEGs recordings were visually analyzed with monopolar montages, the discharges and the artifact were determined by direct following of the recordings, background activity being analyzed during the recording to identify any detectable
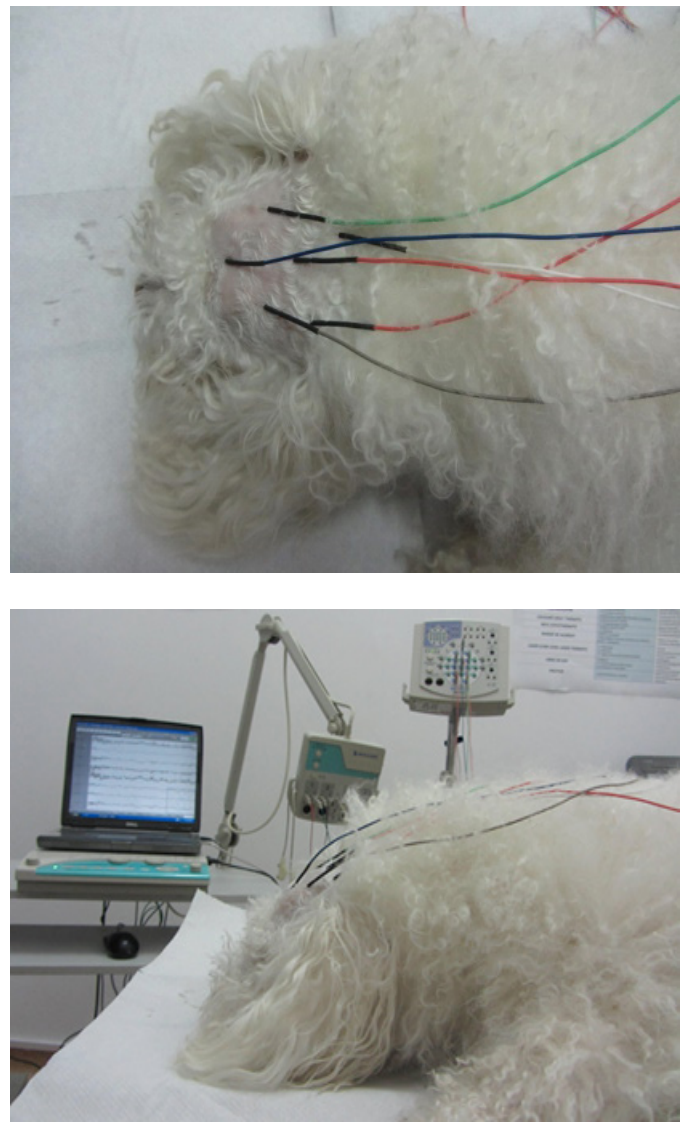

Fig. 1. The Redding model of placement of subdermal needle electrodes 
changes. Recording section was visually selected for analysis of background activity using Fast Fourier Transformation (FFT). Spectral bands were, $8.0-13.0 \mathrm{~Hz}$ for alpha, $13.0-30.0 \mathrm{~Hz}$ for beta, 0.5-4.0 Hz for delta and 4.0-8.0 for theta activity. In order to minimize errors through different skull sizes, forms and thicknesses, the relative power of the spectral bands was calculated for every lead.

\section{RESULTS AND DISCUSSIONS}

Regarding the clinical aspects, all patients showed typical signs of EG poisoning, and were presented for examination at different times after

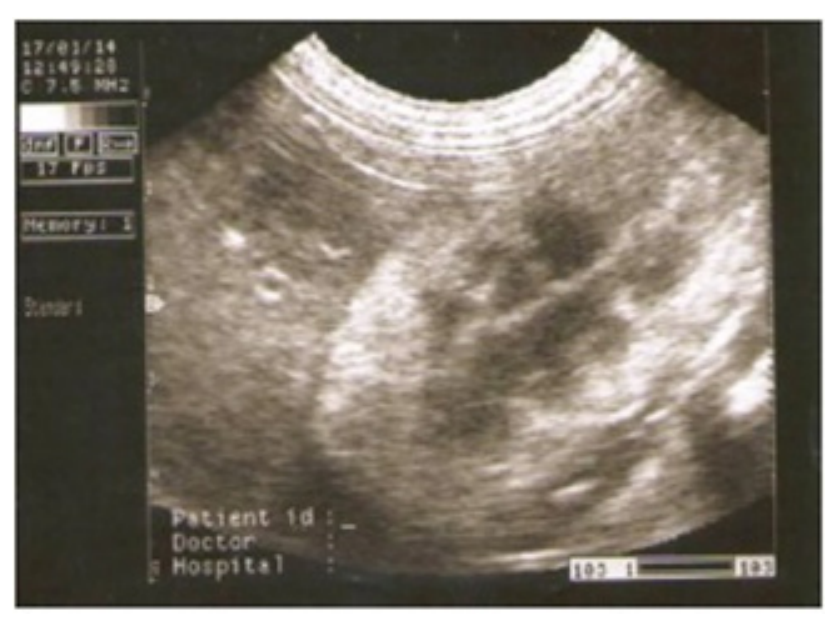

Fig. 2. Hyperechoic renal parenchyma in EG poisoning, Beagle female, 8 years ingestion; only one case was presented within the first 3 hours after consuming the poison, the other 4 dogs after 12 to 24 hours.

Neurologic examination was performed on all patients showing ataxia, decreased reflexes, and seizures-seen in one patient. The blood work completed on all patients revealed high levels of creatinine and BUN, phosphorus and hypocalcemia and marked anionic gap; normochromic, normocytic, non-regenerative anemia, leukocytosis and lymphopenia. Urinalysis was consisting with the suspected diagnosis, showing isosthenuria and envelope forms of calcium oxalate dihydrate crystals, crystals that were seen only in three cases.

Abdominal ultrasounds of the patients sustained the suspicion of EG poisoning showing hyperechoic renal parenchyma.

In the early stages of EG poisoning there was found a normal bioelectrical brain activity, observed on the patient presented in the first 3 hours after ingestion (Fig. 3). As clinical signs advanced, the frequency of spontaneous rhythms decreased and the amplitude increased (13).

Brain activity of all patients was characterized by non-specific electroencephalographic abnormalities: increased percentage of slow wave and high amplitude bursts of delta activity

(10). No interictal epileptic discharges (characteristic for IE condition) were observed.

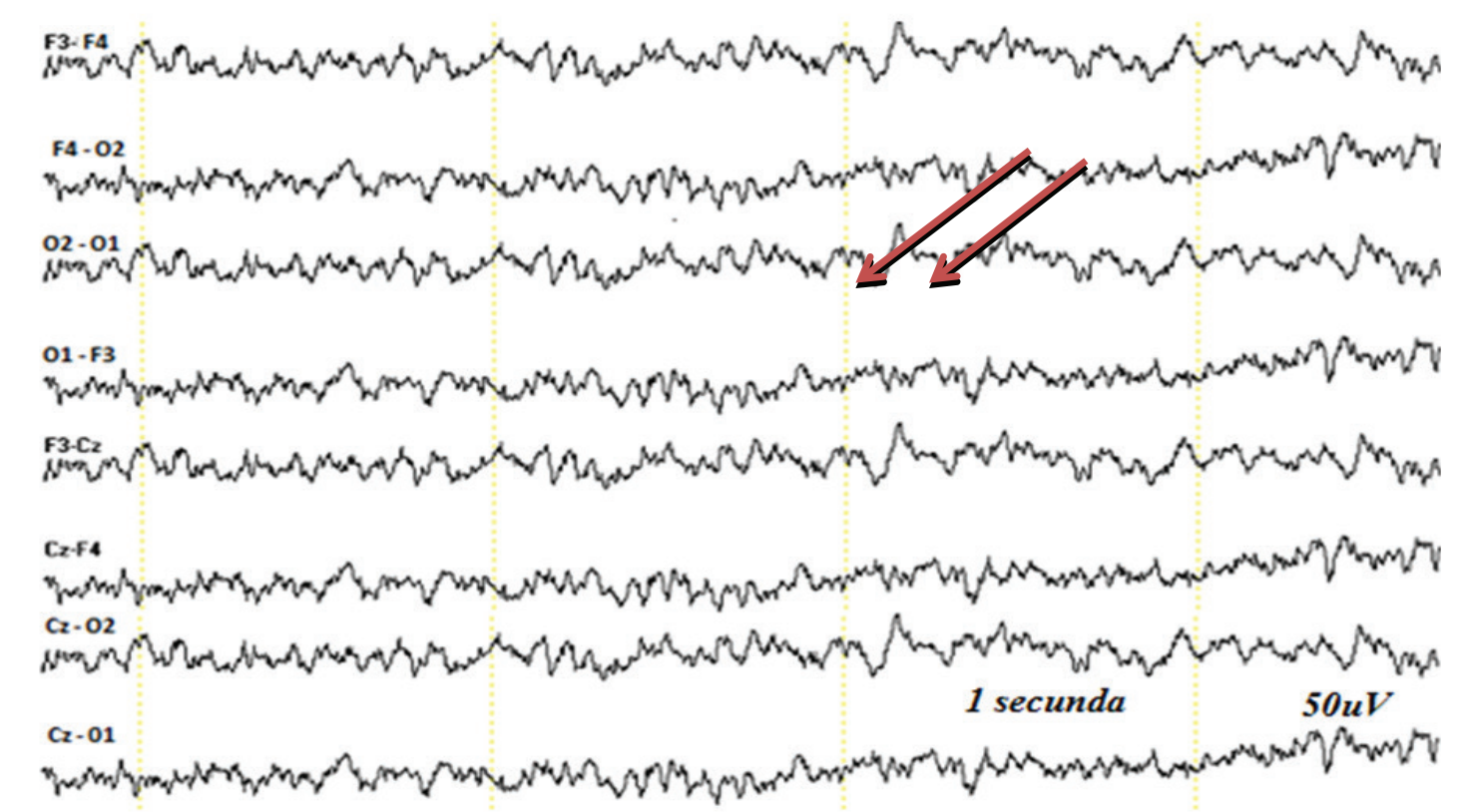

Fig.3. Physiological EEG trace in the early stage of antifreeze poisoning. Delta rhythm and rare theta slow waves. Dog, mixed breed, 4.5 years 
Background activity was characterized by high amplitude and low frequency, probably as a consequence of the use of medetomidine, which corresponds to the studies developed by Hossman et al. (1980), Wallance et al. (1982), Toure et al. (1985) and by Short et al. (1992), who describe the inhibitory effect of alpha-2-adrenoceptor agonists anesthetics on high frequencies $(8,11,14,16)$.

The EEG routes slightly voltated presented an initially slowed background activity of 5-7 cycles/ second, above which synchronous bilateral diffuse delta waves where registered, the expression of

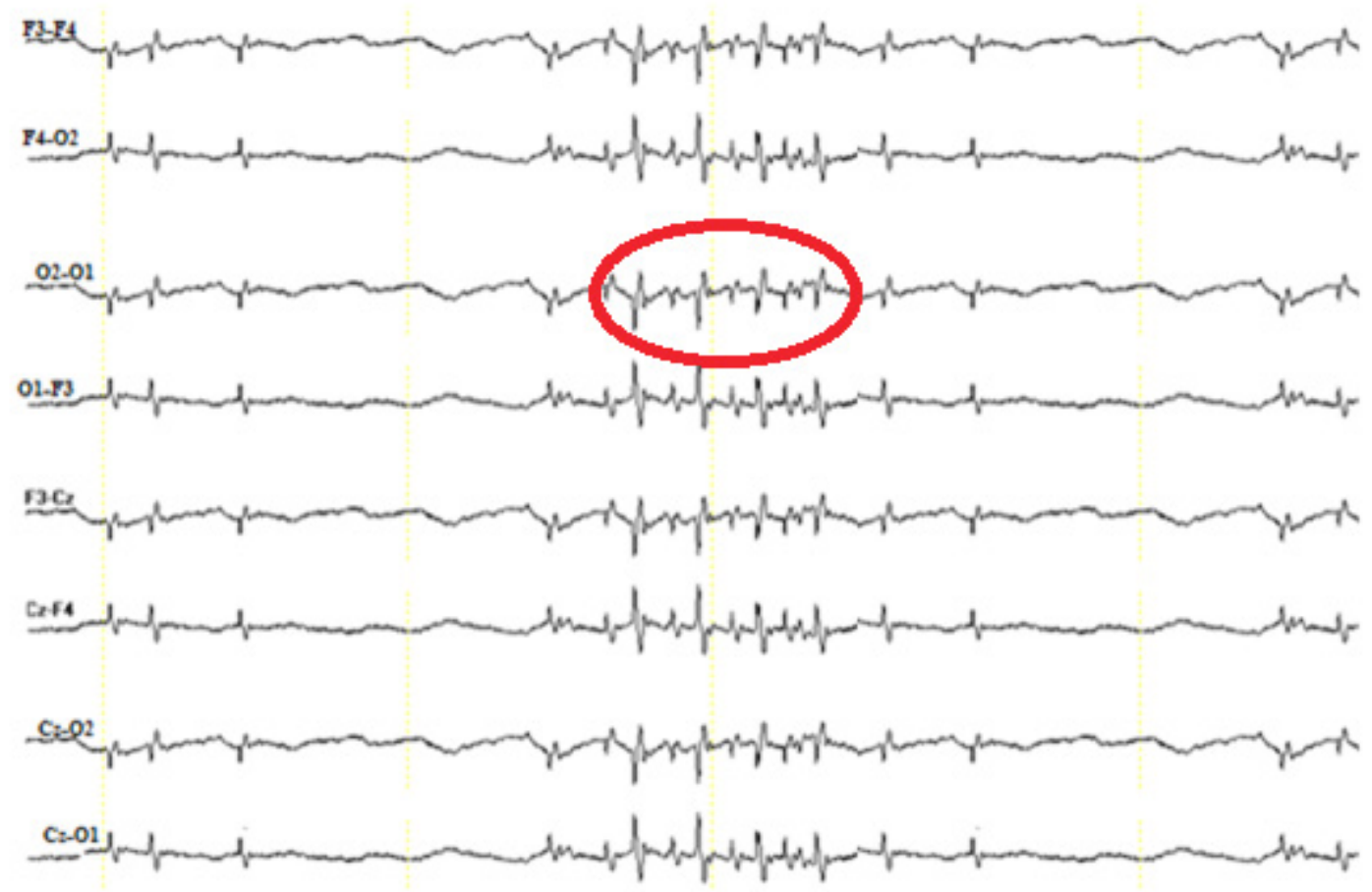

Fig. 4. EEG characterized by synchronous bilateral diffuse delta waves, Beagle, female, 8 years

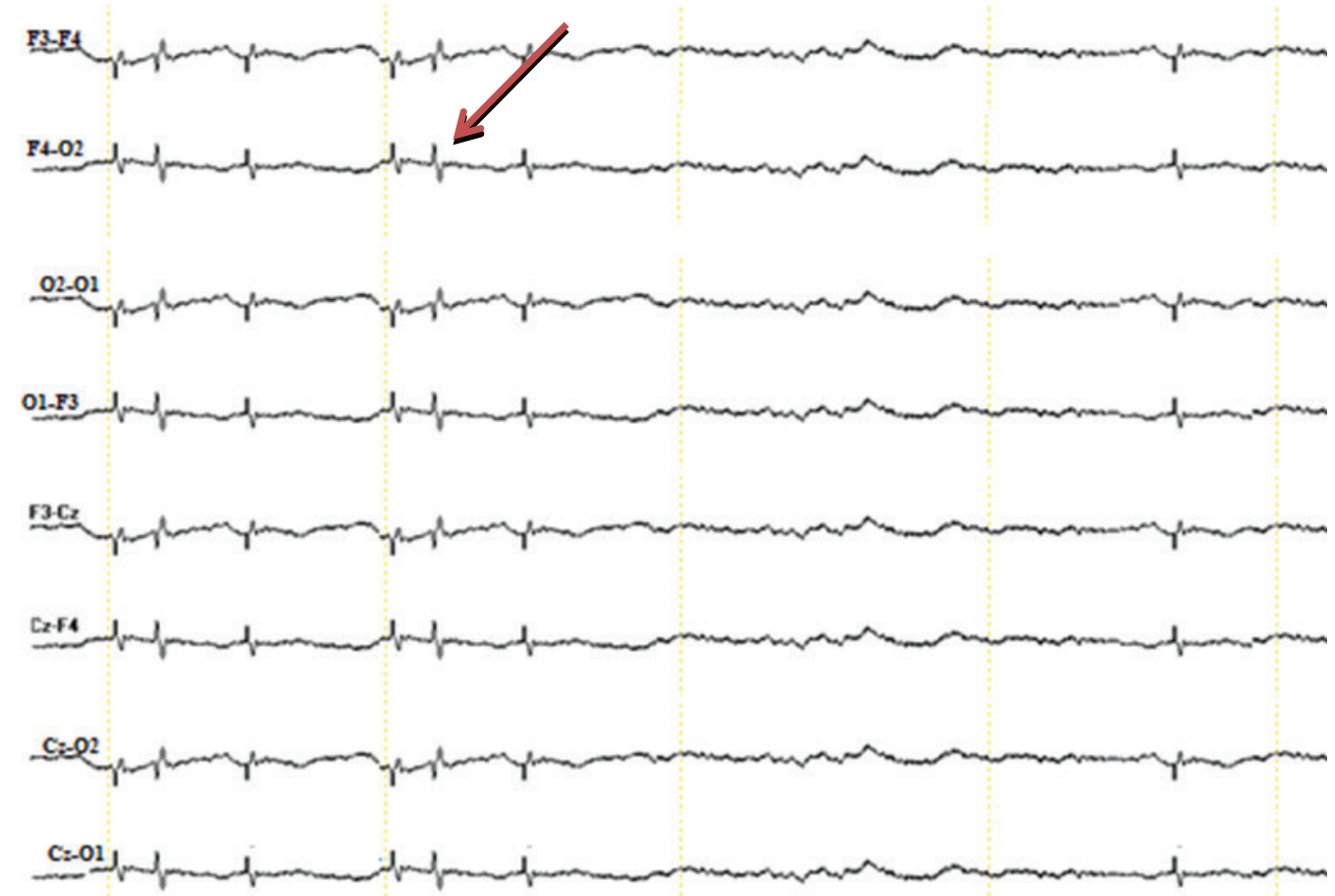

Fig. 5. Sharp and slow wave amplitude reduction routes electroencephalographic 
cortical or subcortical neuronal hyperexcitability state (Fig. 4) .

In advanced stages of EG intoxication the EEG recording route became irregular and arrhythmic with epileptic abnormalities (Fig. 5), clinically manifested before sedation.

These EEG characteristics are seen after 24 hours of ingesting EG (Fig. 5). Highly synchronized slow waves of 1-2 cycles/second (c/s) with a amplitude of $100-200 \mu \mathrm{V}$, appeared in short runs or continuously, which are a self-sustaining expressions of highly synchronized neuronal processes with different synaptic delays (3).

Their occurrence is given by the metabolic and waste products that appear as a result of reduced filtration capacity of the kidney $(5,9)$. In 36 to 48 hours after the poisoning, sharp waves are monophasic, and have a particular morphology with steep upward slope, and the downward slope more inclined which are self-sustaining expressions of highly synchronized neuronal processes with different synaptic delays (Fig. 6).

On the intraictal route, which means during a seizure, the electrical crisis suddenly appeared on all leads, and then intensified by the phenomenon of neuronal recruitment in 2-3 seconds. EEG abnormalities took over on all brain areas and became synchronous bilateral discharges. This is when the whole brain activity adapted to the rhythm developed by the epileptic focus (6). The seizure is characterized by a series of peaks with a frequency of 15-35 cycles/second (Fig. 6), which increased gradually in amplitude, reaching values as high as $250-500 \mu \mathrm{V}$.

Alterations of the bioelectrical activity of the brain is caused by a cumulus of factors, such as the cerebral edema and deposition of calcium oxalate crystals in the walls of small blood vessels in the brain (fig.7), but also the calcium content in the cerebral cortex increases due to the hyperparathyroidism associated with kidney failure (5).

Other toxic metabolites dosed from the cerebral spinal fluid such as guanidine compound guanidinosuccinic acid, guanidine, and methylguanidine that have been identified to be neurotoxic, appear at an elevated level due to impaired kidney function $(4,9,15)$.

Studies have showed that even though BUN is found in high concentration in the serum it does not have by itself a role in the development of the uremic encephalopathy, being considered a surrogate marker for the level of other uremic toxins (15). This excess of matabolites, which alter the brain`s activity in the EG intoxication can be removed through hemodialysis.

Although the proper treatment and antidote was administered to all the patients, four of the five dogs did not survive. Subsequently necropsy revealed pulmonary edema, gastritis,

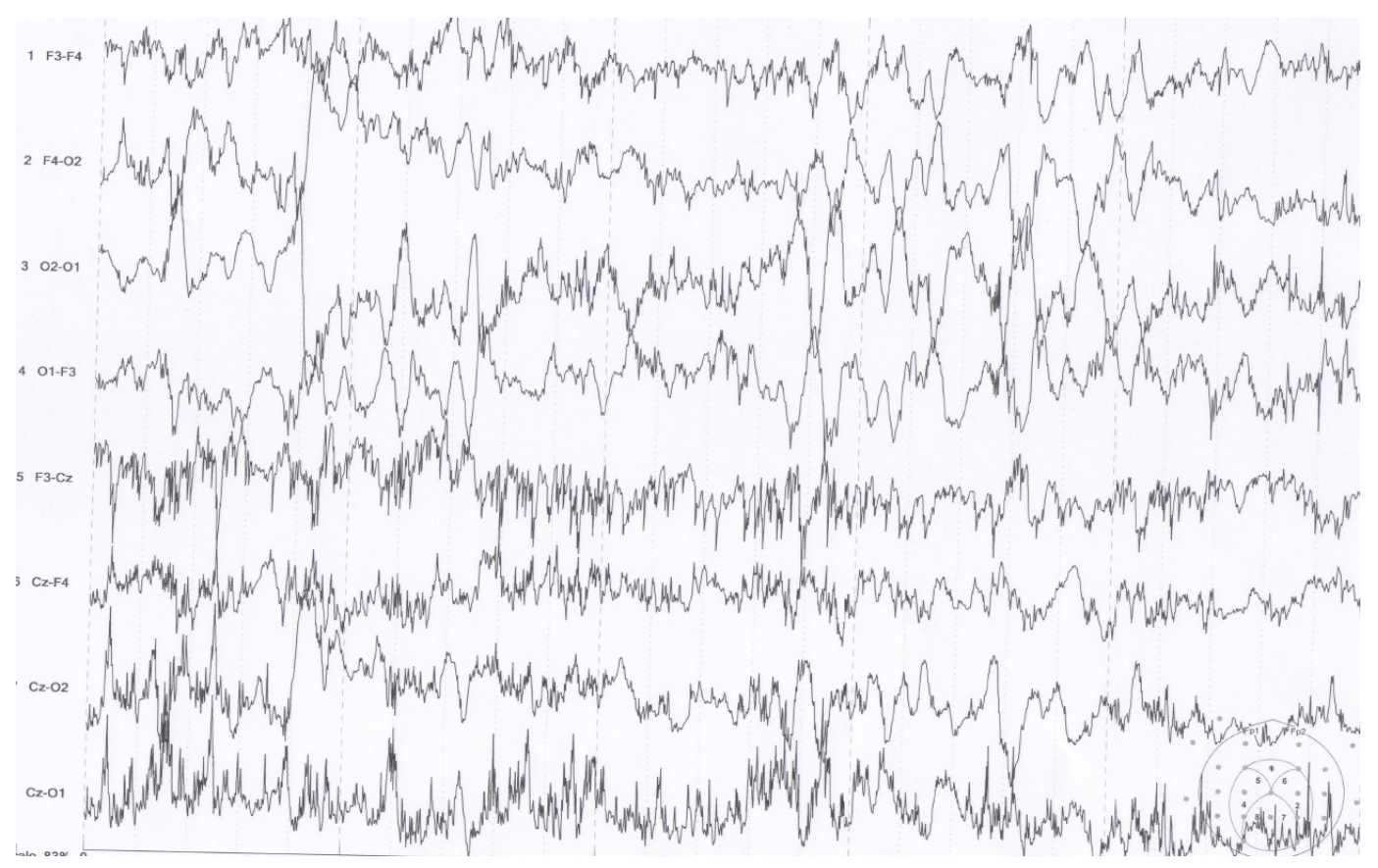

Fig. 6. Intarictal route, epileptiform seizures. Beagle, female, 8 years, advanced stage of EG poisoning 

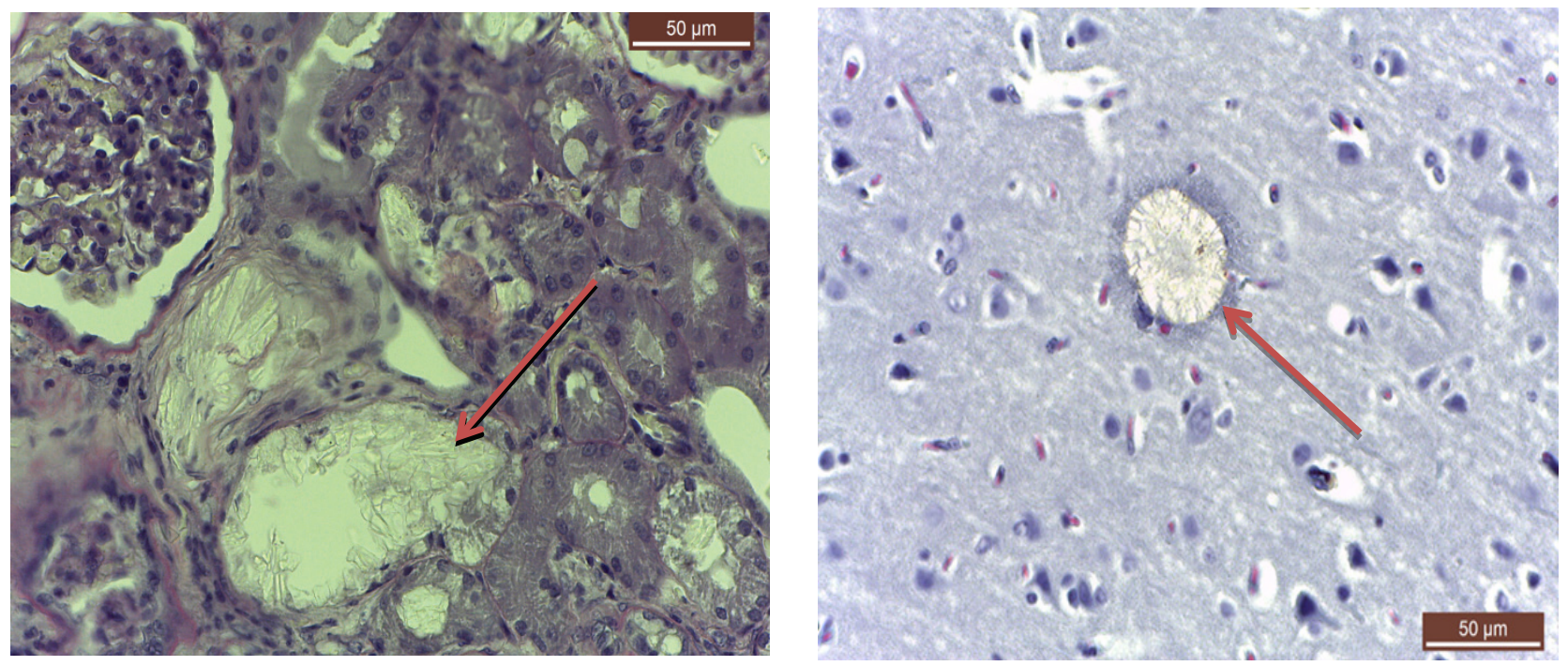

Fig. 7. Calcium oxalate crystals in the renal tubule and in the cerebral cortex

interstitial nephritis, calcium oxalate crystals in the renal tubules and cerebral cortex, with severe degenerative lesions, findings consisting with the initial diagnostic (10).

\section{CONCLUSION}

In the case of EG toxicosis the EEG is characterized by a nonspecific trace, but it can definitely be differentiated from the abnormal bioelectrical activity from idiopathic epilepsy, and also reflects the progression of the neurologic complications associated with the poisoning and subsequent kidney failure.

Even though in early stages of this condition patients do not exhibit alteration of brain activity, gradually EEG abnormalities take over on all brain areas and become synchronous bilateral discharges

The neurologic signs clinically manifested and diagnosed using short time EEG recordings, are the result of toxic metabolites accumulated in excess, especially guanidine compounds, which are toxic for the nervous system causing seizures, and also of the histological alteration of the brain tissue such as edema and congestion.

\section{REFERENCES}

1. Aiello Susan, Mosses MA (2006). The Merck Veterinary Manual, 8th edition, Merial, Philadephia.

2. Aminoff MJ (2005). Electroencephalography: General Principles and Clinical Applications in Electrodiagnosis in Clinical Neurology, Elsevier, Philadelphia, 37-84.

3. Brounds R, De Deyn PP (2004). Neurological complication in renal failure: a review. Clinical Neurology and Neurosurgery 107:1-16.
4. Burn D J, Bates D (1998). Neurology and the kidney. Journal of Neurology, Neurosurgery and Psychiatry 65:810-821.

5. Dumitru D (1995). Electrodiagnostic medicine, Hanley \& Belfus, Philadelphia, 29-64.

6. Eder Anne F, Cindy M McGrath, Yvonne G Dowdy, Tomaszewski, J E, Frances M Rosenberg, Wilson RB, Wolf BA, Leslie M Shaw (2004). Ethylene glycol poisoning: toxicokinetic and analytical factors affecting laboratory diagnosis. Clinical Chemistry 44:168-177.

7. Ettinger SJ, Feldman AC (2004). Textbook of veterinary medicine. Intoxication versus acute, nontoxicologicilnesses, the differenatation of the two, Elsevier Saunders, 2-242.

8. Hossman V, Maling TJB, Hamilton CA, Reid JL, Dollery CT(1980).Sedative and cardiovascular effects of clonidine and nitrazepam. Clinical Pharmacologyand Therapeutics 28, 167-176.

9. Klaus K (2002). Metabolic encephalopathies, Journal of Neurology, 249:1150-1159.

10. Pașca SA, Slocan Gh, Șindilar EV, Lazăr M (2010). Clinical and morphopathological aspects in anti-freeze intoxication of dogs, Scientific Works USAMV Bucharest, series C, 58 (1-2):296-305.

11. Short CE, Raiha JE, Raiha MA, Otto K ( 1992). Comparison of neurologic responses to the use of medetomidine as sole agent or preanesthetic in laboratory beagles. Acta Veterinaria Scandinavica 33:77-88.

12. Solcan Gh ( 2011). Bolile rinichilor. Medicina internă a animalelor,Eurostampa, Tmișoara, vol 2:24-71.

13. Steinke W, Arendt G, Mull M, Reiners K, Toyka KV (1989). Good recovery after sublethal ethylene glycol intoxication: serial EEG and CT findings. Journal of Neurology 236:170-173.

14. Tourai K, Senba H, Sasaki N, Ohashi F, Takeuchi A, Usui $\mathrm{K}$ (1985). Developmental EEG of the beagle dog under xylazine sedation. The Japanese Journal of Veterinary Science 47: 459-463.

15. Vanholder R, Glorieux G, DeSmetR, Lamier N (2003). New insights in uremic toxins. Kidney International Supplement 84:6-10.

16. Wallance BP, Lawrence GS, Vaikunth NP (1982). Morphinelike effects of clonidine on the EEG, slow wave sleep and behaviour in the dog. European Journal of Pharmacology 81:551-557. 